\title{
A chorus learning support system using the chorus leader's expertise
}

\author{
Mizue Kayama $^{1, *}$, Kazunori Itoh ${ }^{1}$, Kazushi Asanuma ${ }^{2}$, Masami Hashimoto ${ }^{1}$, Makoto Otani ${ }^{1}$ \\ ${ }^{1}$ Department of Informational Engineering, Faculty of Engineering, Shinshu University, Nagano, Japan \\ ${ }^{2}$ Nagano Prefectural Institute of Technology, Ueda, Japan
}

\section{Email address:}

kayama@cs.shinshu-u.ac.jp (M. Kayama)

\section{To cite this article:}

Mizue Kayama, Kazunori Itoh, Kazushi Asanuma, Masami Hashimoto, Makoto Otani. A Chorus Learning Support System Using the Chorus Leader's. Science Innovation,. Vol. 1, No. 1, 2013, pp. 5-13. doi: 10.11648/j.si.20130101.12

\begin{abstract}
The purpose of this study is to explore a chorus learning support environment based on the tutoring knowledge of the chorus leader. In general chorus practice, a chorus leader tends to instruct his chorus members based not on score information, but on a chorus leader's sense of value and/or his musical philosophy. We try to extract the tutoring knowledge necessary to evaluate/review a singing voice from the chorus leader. The extracted knowledge is expressed in a computational form and implemented in a learning support system. In this paper, a chorus learning support system is proposed. At first, the tutoring knowledge of a chorus leader is described. Next, the architecture and functions of the proposed system are shown. Finally, we evaluate the adequacy of the extracted knowledge and the effectiveness of chorus learning with our system.
\end{abstract}

Keywords: Learning Support System; Performance Support; Chorus; Chorus Leader's Expertise; Singing Voice

\section{Introduction}

Many of us have experienced singing in a chorus. The main features of a chorus are "Singing in a group", "Singing several parts", and "Singing in harmony over all parts and/or in a part". At a typical chorus lesson, not only singers, but also an accompanist, a conductor and/or a leader are joined in one group. The leader, who in some cases also serves as the conductor, is responsible for coordinating the whole choir activities. In this study, we define a singer as a learner, and the leader as a tutor/instructor.

In general, chorus learning with a leader is done as follows:

(1) learners sing some phrases as instructed by the leader.

(2) the leader gives some advice/guidance to the learners during and/or after their singing.

(3) learners sing again making the requested changes in (2) in his/her own way.

These three steps are repeated until the leader judges that the whole song is properly sung by this chorus group. As a result, the harmony of the chorus group is closer to the ideal style of the leader. At the same time, the vocal skills of each individual learner are improved.

\section{Related Work}

In typical chorus learning, a leader gives some advice for singing a song, e.g., "Let's sing with a smile", then learners sing a song or some phrases. After singing, a leader gives some instructions, e.g., "Smile more" or other advice, e.g., "Be careful at the first note in the next phrase", to the learners, and the learners sing again. Each learner uses a process of trial and error to make their singing voice perfect. Therefore, chorus learning is said to be a kind of skill learning.

Skill learning is a kind of learning accompanied by body movements. In singing learning activities, a singer needs to change his/her voice consciously, by controlling his/her vocal organs. This act is accompanied by an interaction of verbal interpretation and physical movement, so it relates to skills involved in human cognitive processes. These types of skills are known as physical skills. In particular, the chorus is a group, so others (members of the same part and/or members in other parts) need to be aware of each other. Therefore, a chorus needs more open and advanced physical skills.

In recent years, a variety of learning environments to develop personal skills has been explored [1-3], e.g., drawing [4] or playing sports (karate [5] / baseball [6]). In 
these types of learning, learners try to improve their skills while imitating the action of professionals and experts.

Meanwhile, in a chorus, the learning goal is to try to push the singing voice closer to the ideal harmony and musical philosophy of the leader. In chorus practice, leaders give advice to improve a singer's voice not on score information, but a chorus leaders' sense of value and/or his musical philosophy. Each leader has his/her own training method, so chorus learning styles and methods vary among different leaders $[7,8]$. In other words, the chorus leader is of paramount importance in a chorus lesson.

The leader plays quite an important part to create chorus songs. There are a few case studies and research that focuses on the role of the chorus leader [9]. At chorus practice, a learner comes face-to-face with a leader and/or other chorus members. Therefore, time and place constraints are raised. To support the chorus training/practice, if an appropriate learning environment is arranged by using ICT on behalf of the human chorus leaders, learners are able to try to improve their personal skills anywhere by themselves.

There are two main methods to support singing practice. One method is based on theory. Learners read and/or listen to the learning materials to absorb knowledge of singing. The other method is based on experience. Learners take part in problem-solving activities about singing. In particular, in previous studies on the latter method, some singing support systems named SINGAD [10], ALBERT, SING \& SEE and WinSINGAD [11] have been proposed. Real-time visual feedback about the pitch / spectral ratio / amplitude / timbre (spectrogram) is presented by these systems. These studies report that visualizing the difference between the ideal pitch and the learner's pitch contributes to improvement in accuracy. MiruSinger [12] is able to give visual feedback in synchronization with the vocal pitch of the learner and a professional singer's pitch. This research reports that by using this methodology, a learner can improve his/her vocal ability and understanding of singing. Moreover, commercial software for choir practice has been announced [13].

All these systems focus on reducing the differences in the learner's vocal pitch and the ideal pitch which is shown in a musical score or a professional singer's voice. However, in this study, the musical philosophy and/or the performance interpretation of chorus leaders is focused on and described in a computational expression. We extracted and formulated the expertise of chorus leaders. Based on these results, we propose a chorus learning support system using the chorus leader's tutoring knowledge.

\section{Chorus Leader and Singing Voice Evaluation}

We continuously observed one chorus group, which was instructed by a particular chorus leader. From this, the evaluation strategies for singing and instruction methods for inadequate singing were collected $[14,15]$. In this section, the expertise of a chorus leader is studied based on these results.

\subsection{Classification of the Expertise}

In giving instruction to chorus members, it is important to consider the personality of the chorus group and/or the musical views and interpretations of the chorus leader. In this study, we define these kinds of knowledge as expertise of the chorus leader. The categories are application phase, application data and application scope. Application phase means pedagogical activities by the leader. Subcategories are evaluation and instruction. Application data corresponds to voice information. Subcategories are acoustical features as physical quantities, and interpretation of the singing as psychological quantities. Application scope means the difference in expertise between leaders. Leaders' expertise differs according to each leader's musical views and experience. Therefore, the scope is divided into specific expertise and common expertise.

\subsection{Chorus Leader}

In this study, we adopt both evaluation phase and instruction phase as the application phase, acoustic information as the application data, and lump knowledge (common and specific knowledge) as the application scope. The chorus leader which has that expertise is a professional chorus master. He has been a chorus conductor for 29 years, and is a member of the Japan choral directors association. His learners are all in chorus groups in a local community. The learning aim of chorus members is to improve their literacy with skill development [16]. This is one method of non-formal learning. The chorus song which we analyzed (Song name: YORU NO UTA, Lyrics: Norio Sakata, Music: Sasaki Nobuhisa) was arranged by this master.

\subsection{Acoustic Features of a Singing Voice}

In the musical score for a chorus, there is much musical information. For example, the rhythm of each phrase and the whole song, breathing position, pitch, vocal volume, phonetic values, and lyrics. In our study, we evaluate the score, pitch, power, phonetic values and rhythm. These can be calculated as physical values. Meanwhile, from the singing voice, the pitch (fundamental frequency), power, spectral structure and the singer's formants can be extracted. Furthermore, from these changes in time, note values, rhythm and vibrato can also be calculated.

Pitch, power, note values and rhythm can be related to the information extracted earlier from the musical score. However, the spectral structure and singer's formants cannot be related to the objective factors. Therefore, in this study, pitch and power, which have higher priority as acoustic features, are used to support chorus learning. These factors are easily extracted from both singing voice and musical score.

In the proposed system, these factors are used as an 
indicator for the evaluation of a singer's voice. The system calculates the differences in this information in the singing voice and score. These differences are used for real-time and post-singing instruction.

There are two types of representation for pitch. One expression is based on intervals between two notes, and the other is based on frequency. We call the former "cent" and the latter "Hz". In this study, we use the cent scale for pitch expression.

\section{Expertise for Chorus Training}

\subsection{Priority of Instruction}

In our previous research, we identified the elements of the singing voice necessary for evaluation/instruction. They are power (volume), pitch, timbre, phonetic value and rhythm[9]. The weight for each element is related to the priority order in instruction. The priority order of that master is 1.pitch, 2.power, 3.phonetic value, 4.tone and 5.rhythm. The leader pointed out that pitch, power and phonetic value are affected by a singer's vocal sound. On the other hand, tone and rhythm are affected by the chorus leader's instruction. He also pointed out that this priority order could be changed according to the singer's learning level.

\subsection{Singing Voice Evaluation for One Note}

\subsubsection{Pitch Evaluation}

When a chorus leader evaluates the pitch of a singing voice, he tends to make allowances for a permissible zone to judge correct pitch. We extract the permissible zone from the master. He evaluates some singing voices using the same phrases. Each voice includes some off-pitch notes. The evaluation method is a five level evaluation, so that 5 means "quite correct" and 1 means "terrible". Experimental results are shown in Fig.1. The horizontal axis shows the relative pitch, and the vertical axis shows the evaluated score. Each point expresses an evaluated voice. In the evaluation score 5 and 4 are acceptable levels. The acceptable voices are distributed around the ideal pitch in a range of plus or minus 25 [cents]. This result was confirmed in other experiments. Based on these results, the range of the permissible zone for pitch for this master is found to be plus or minus 25 [cents] (one quarter-tone).

\subsubsection{Power evaluation}

By the same experiments as in 1), we were able to extract the expertise about power evaluation. There was no relationship between the dynamic marks on the score and the evaluated results by the master. However, some distinctive changes in power show clear correlation between the evaluated results. These changes are the overshoot phenomenon shown in Fig.2. The overshoot phenomenon is a momentary fluctuation when the singing power increases soon after vocalization, then shortly after that decreases quickly to a relatively constant value. This phenomenon is identified in $80 \%$ of incorrect data and in $40 \%$ of correct data. Based on this result, the occurrence and/or frequency of this overshoot phenomenon shows the influence on the evaluated results by the master. We define the occurrence of this phenomenon as one of the expertise for chorus training. As evaluation points for the volume of a singing voice, the master suggests that the important points are whole volume balance and continuous and smooth changes in volume. The overshoot phenomenon can cause losses in both continuity and smooth changes in volume. Therefore, singing data which have some overshoots are judged as incorrect voices.

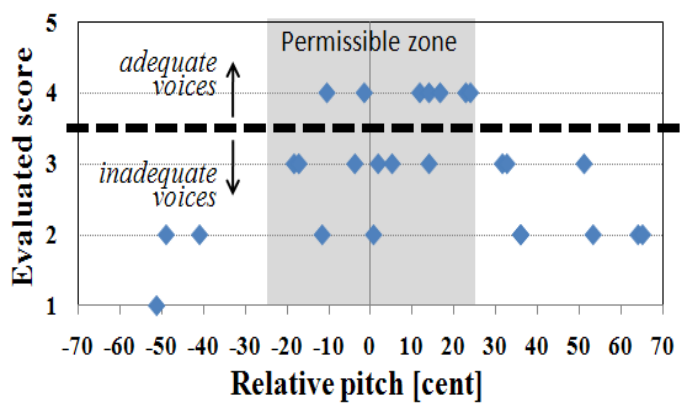

Figure 1. Relation between the relative pitch and the evaluated score(D\#2).

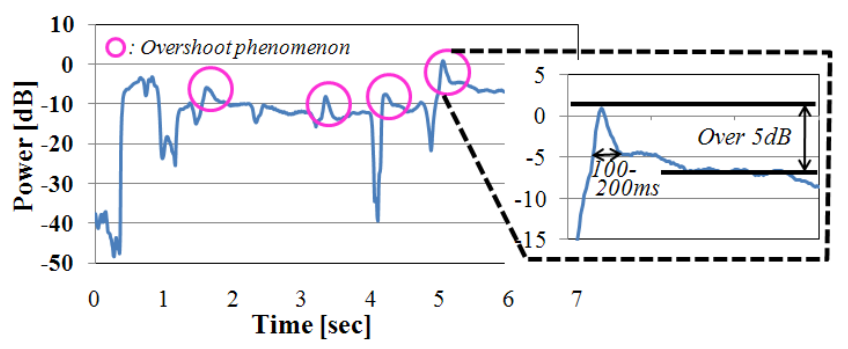

Figure 2. Overshoot phenomenon of volume in a singing voice.

\subsection{Singing Voice Evaluation for Some Notes}

In an actual chorus lesson, learners often practice specific phrases of a song repeatedly before they sing through the whole song. In this study, we call this "phrase practice".

We extract the knowledge necessary to evaluate the learner's singing voice in phrase practice from a choral leader. In this experiment, the leader is asked to evaluate some singing data during phrase practice. He judges the instruction necessary to improve the learner's voice. The leader also describes the negative aspects of singing voices which require improvement.

The subject is a male choral leader, who has instructed some choral societies for 18 years. The chorus song which we analyzed in this experiment was arranged by this subject. This song was the same as the song which was explained in 3.2. Two phrases with four-bar in this song (Phrase-A, Phrase-B) were selected as evaluation phrases. These phrases were determined based on the opinions of the chorus leader. If a novice singer sings these phrases, it is easy for the leader to judge the ability of the singer.

Nine males in their 20s, who were choral beginners with 
no experience with formal choral or vocal lessons, sung the phrases and their singing voices were recorded for use as test data. A total of 17 sets of data were collected and used. For the singing data in Phrase-A (9 data), 3 data were judged to be inappropriate voices. For Phrase-B (8 data), 2 data were judged as inappropriate. The negative aspects of these data are as follows:

- $\quad$ the overall tone shifted.

- a particular note had extremely low pitch.

- $\quad$ the pitches of all notes were very unstable.

- $\quad$ in more than half of the notes in this phrase, their pitches were off.

Based on the above results, we hypothesize that the evaluation criterion for phrase singing are the following three points.

(1) pitch instability.

(2) average offset between the ideal pitch and the pitch of the singing voice for all notes in a phrase.

(3) ratio between the number of all notes in a phrase and the number of sung notes that differ more than the permissible difference from the ideal pitch.

To represent the first item, we analyze the difference between the ideal pitch and the average pitch which is calculated in every half time of the shortest note in the song. In this study, "Pitch instability" means that these differences show the movement from negative to positive or positive to negative, and each difference is more than +25 [cents] or -25 [cents]. For the second item, based on the results of the experiment described above, if there is a plus or minus $50-60$ [cents] disparity in average offset, the leader judges his/her phrase sing to be inadequate. For the third item, based on the results of above experiment, we find the permissible difference is less than plus or minus 50 [cents]. Moreover if the ratio of notes which are out of the permissible zone is more than $50 \%$, the leader judges his/her phrase singing to be inadequate.

But there is one datum which does not fit any of the above criteria. This is due to other acoustic features that may potentially influence the evaluation. Extraction of that kind of knowledge is a topic of future work.

\section{Validation of Extracted Expertise}

\subsection{Instructional Expertise for One Note}

In this section, we want to verify the appropriateness of the knowledge extracted from the master. In this validation, the master is requested to judge some singing voices, which are gathered from another novice chorus group. This group has never been directed by the master. The master ranks the voices on a scale of 1 to 10 . We examine the relationship between that rank and the acoustic features of each voice.

Fig. 3 shows the validation results. The horizontal axis is the rank of the evaluated voices. The left vertical axis is the number of notes which differ by more than plus or minus 25 [cents] from the ideal pitch (dark-gray line). The right vertical axis is the number of overshoots in volume (light-gray line). As the rank of the voice decreases, the number of off-pitch notes tends to rise.

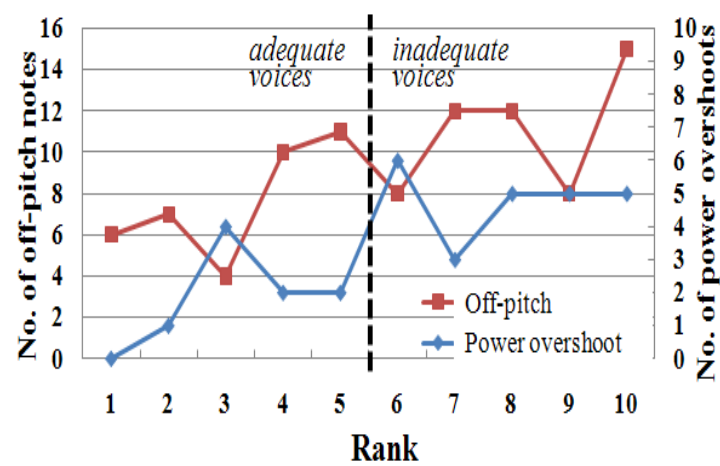

Figure 3. Result of an adequacy evaluation of the extracted tutoring knowledge.

Thus, the extracted expertise that the range of the permissible zone to judge correct pitch is plus or minus 25 [cents] is reasonable. However, the voices with ranks 3 and 6, which have a lower pitch difference than voices in nearby ranks, go against the above trend. It seems that these voices have more volume overshoot than other voices. In other words, first the master evaluates the pitch accuracy, then checks the smoothness of its power. This shows that the tutoring strategies to determine the instruction priority we arranged are reasonable.

Based on these results, by using the expertise we extracted from the master, the evaluation function of our system is able to simulate the voice evaluation of the master.

\subsection{Instructional Expertise for Some Note}

An experiment was conducted to verify the expertise shown in the previous section. We compared the evaluation results of the chorus leader and the results of the automated evaluation by the expertise we mentioned above for the same singing data set. The chorus song which we used in this experiment was arranged by this chorus leader (Song name : Michi, Lylics : Shogo Kashida, Music : Miwa Furuse.).

Two phrases with four-bar in this song (Phrase-C, Phrase-D) were selected as evaluation phrases. These phrases were determined based on the opinions of the chorus leader. The 40 singing data which were sung by six males in their 20s were used for this experiment. They have different experience with chorus practice. Two are novices, two are members of amateur choral societies and two were members of amateur choral societies.

The leader judged 22 data to be adequate voices. By using the three evaluation criteria we mentioned above, we want to express the difference between the data judged to be adequate singing and the data judged to be inadequate singing.

The corresponding data for the first item (Pitch instability) are not included in the evaluated data. Based on 
the second item, the average offset of all singing data are shown in Fig.4. The upper part is inadequate data and lower is adequate. The data distribution in the upper part is more biased toward the right than the lower part. The average of the upper part is 52 [cents], while that of the lower part is 19 [cents]. By using the Student t-test, we can find a statistically significant difference between these two data sets $(p=0.01)$. Thus, this result leads to the suggestion that this leader uses this criterion for evaluation of phrase singing.

Based on the third item, the ratios of the number of sung notes that differ more than plus or minus 50[cents] from the ideal pitch are shown in Fig.5. The upper part is inadequate data and the lower is adequate. The data distribution in the upper part is also more biased toward the right than lower part. The average of the upper part is $49 \%$, while that of the lower part is $18 \%$. Using the Student t-test, we can find a statistically significant difference between these two data sets $(p=0.03)$. Thus, this result leads to the suggestion that this leader uses this criterion for evaluation of phrase singing. However, the variance of the upper data is large and the data distribution is wide. We can use the second criterion after the first item is applied. In conclusion, it is possible to estimate an adequate voice for phrase singing by using the evaluation criterion shown in 4.3 .

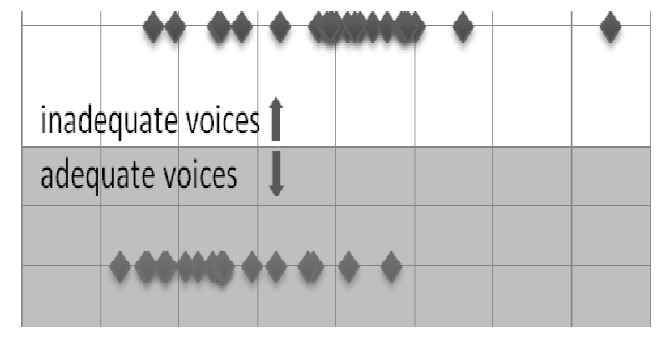

\section{$\begin{array}{lllllllll}0 & 10 & 20 & 30 & 40 & 50 & 60 & 70 & 80\end{array}$ The average offset width [cent]}

Figure 4. The average offset (upper part is inadequate data and lower is adequate).

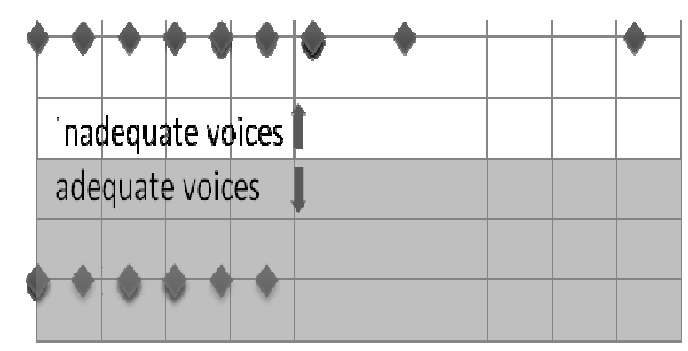

$\begin{array}{lllllllllll}0 & 10 & 20 & 30 & 40 & 50 & 60 & 70 & 80 & 90 & 100\end{array}$

\section{The ratio of notes [\%]}

Figure 5. The ratio of the number of sung notes that differ more than plus or minus 50[cent] from the ideal pitch.

\section{Chorus Learning Support System}

\subsection{Overview}

Our chorus learning system processes a singing voice. The input is a learner's voice. The output is some instruction for the learner based on the expertise, which was described in the previous section. As real-time visual feedback for the learner, our system shows the tracking data of the vocal pitch and volume in a time series. At the same time, the ideal pitches in the score are also presented. Therefore, a learner can visually compare the difference between score pitch and his/her singing results. Also, as real-time auditory information, accompaniment music by MIDI (piano), singing voices of other parts and/or singing voices of his/her own part are presented. The singing voices of other parts are created by using singing synthesizer software [17]. The synchronized singing voices (self-part, any other part, all parts) can be chosen when our system is started.

After a learner sings a song, our system gives two types of instructions about pitch and power.

- $\quad$ some suggestions to improve his/her voice.

- pointing out the phrase or word which needs extra attention (many learners too often make mistakes in that part).

The first item is calculated based on the singing log data of the learner. This function provides personal adaptive learning support. To express the suggestions, some instruction words used by the master during his lessons are applied. As for the second item, the singing log data of all learners which practiced this song are used. In this case, the instruction words are also used to express advice.

\subsection{System Architecture}

The proposed chorus learning support system has knowledge-based evaluation and instruction for singing voices. The tutoring strategies to determine the instruction priority, the allowances for the permissible zone to judge correct pitch, the detection algorithm for the overshoot phenomenon on power and some instruction words gathered from the master are implemented as the expertise for chorus learning in our system.

Fig. 6 shows the architecture of our system. This system consists of three phases, the acoustic feature extraction phase, the evaluation phase and the instruction phase. In the first phase, the pitch and power of the singing voice are extracted from the learner's voice in real-time. In the second phase, based on the score information and evaluation expertise of the master, extracted features are divided into correct parts and incorrect parts. In the final phase, for the incorrect parts in learner's singing, first, the system identifies the priorities to correct, then the worst three singing points are chosen. Based on the expertise extracted from the master, the system gives some instruction to improve the learner's singing. 


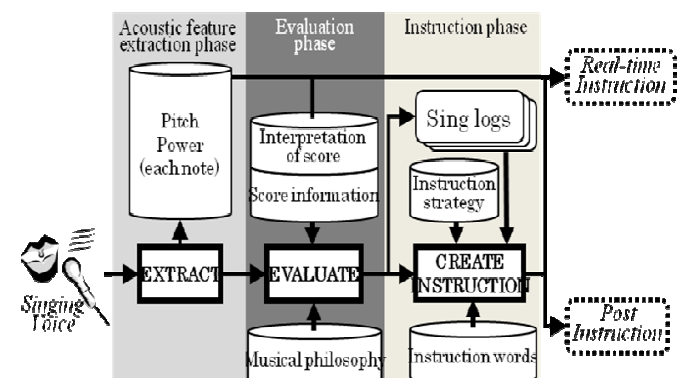

Figure 6. System Architecture.6.3. Real-time and Post Instructions

\subsubsection{Learning Flow}

The learner can be given both real-time instruction and post learning instruction. During his/her singing, the system presents both visual and auditory feedback information to the learner as real-time instruction. A learner can practice his/her chorus song by seeing both ideal pitches in score and his/her own pitch in a time series, and by hearing the synthesized singing voice in his/her own part and/or other parts. After singing, a learner can record his/her most recent results. The system shows the evaluation results based not only on his/her past log data but also other learner's log data. When a learner sings again, in addition to the general real-time feedback, the instructions based on the most recent post-instruction are shown as visual feedback.

\subsubsection{Interface}

Fig.7 shows an example of the screen image during real-time instruction. Four areas are shown:

lyrics and instruction words area.

pitch area.

musical score area.

power area.

Here are the roles of each area.

1) Lyrics and instruction words area : In this area, lyrics related to the displayed phrase are shown(II). If a learner has been given the post instruction, some instruction words which are related to a particular phrase are displayed(I). Then shortly after his singing, an evaluation result (correct or not) is also shown. In addition to the above information, the system displays the poorly sung part (single Japanese character) in the lyrics in red letters (IV, "NO"[in Japanese]).

2) Pitch area : In this area, the ideal pitch series and the learner's pitch series are presented simultaneously. The sampling rate for the voice is chosen by learner when the system starts. The vertical axis shows the pitch value (either $\mathrm{Hz}$ or cent). The score pitch is expressed by green lines, and for the poorly sung parts, a red line is used. The learner's pitch series (shown as points) are overlaid on the score pitch. At this time, red points correspond to incorrect parts, and blue points correspond to correct parts. For parts where this learner has sung off-pitch in the past, an alert message (IV) is displayed by the system.

3) Musical score area : In this area, the musical score of the singer's part is displayed in a time series. Breathing positions are also indicated.
4) Power area : In this area, the power of the learner's voice is displayed in a time series. The vertical axis shows the vocal power $[\mathrm{dB}]$. In addition, the occurrences of the overshoot phenomenon are indicated in this area $(\mathrm{V})$.

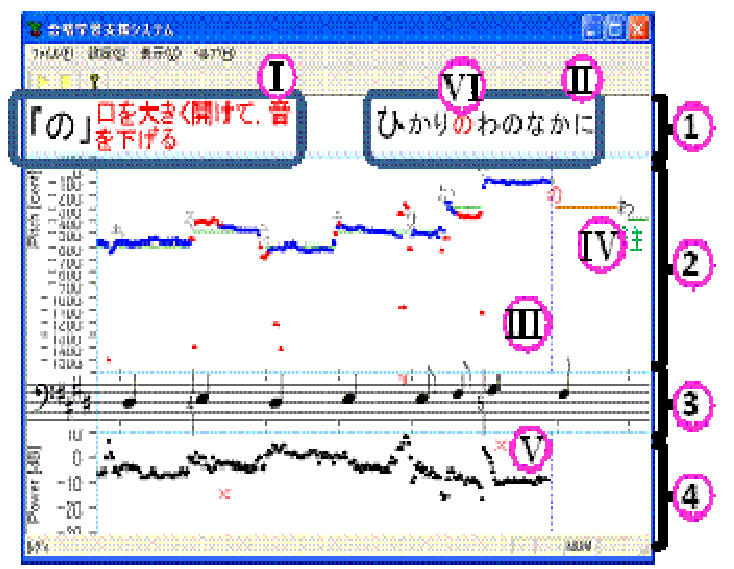

Figure 7. An example screen image of the system interface for real-time instruction.

\subsubsection{Post Instruction}

Fig.8 shows an example screen image of the post instruction. Six types of instructions are displayed: the pitch and power evaluation for the previous instruction ("Good Job" means corrected, "Not Good" means not corrected), the number of off-pitch parts, the number of occurrences of the overshoot phenomenon, the total singing score, the worst three parts in terms of pitch and power and the instruction words to correct these worst parts.

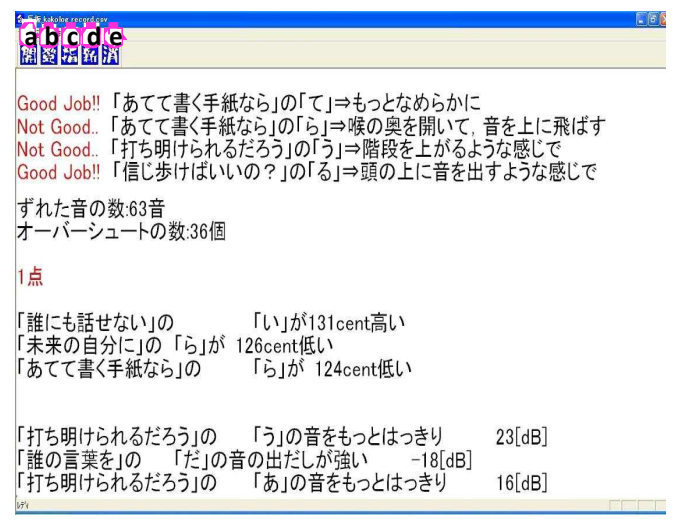

Figure 8. An example screen image of the system interface for post instruction.

\subsection{Phrase Practice Support Function}

To make our chorus learning support environment more sophisticated, a supporting module for phrase practice based on the expertise we described in Chapter 4 was added.

Fig.9 shows the interface for phrase choice and giving singing instruction. Fig.10 shows the interface for phrase practice. This function works with the real-time instruction we mentioned in 6.3. During phrase practice, the same auditory information as for whole song practice is 
presented to the learner. When using this function, a learner can choose any phrase in the song. With this function it is possible to evaluate changes in voice pitch and volume of the chosen phrase in real time. When a learner uses this function, our system stores the evaluated results of his/her singing voice in a log. Therefore, this function can judge a learner's singing voice in a comprehensive manner based on data of his/her past singing. Moreover, identification of where errors occur repeatedly and estimation of the type of errors are possible with this function.

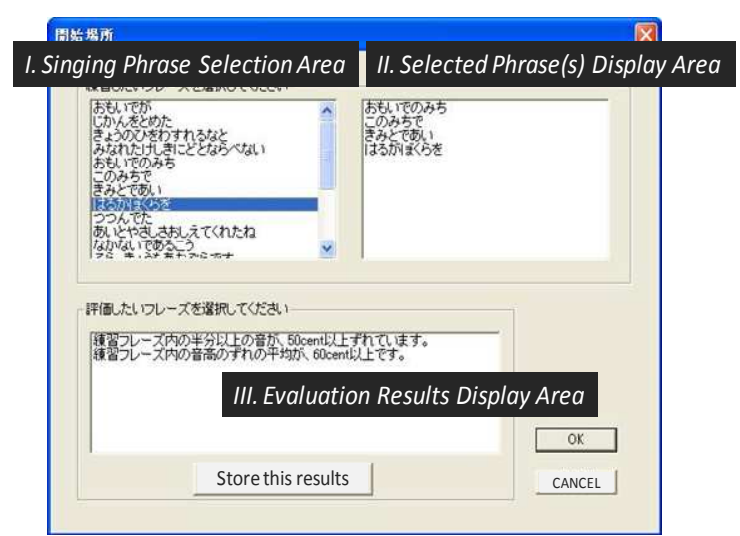

Figure 9. An example screen image of the system interface for phrase selection dialogue.

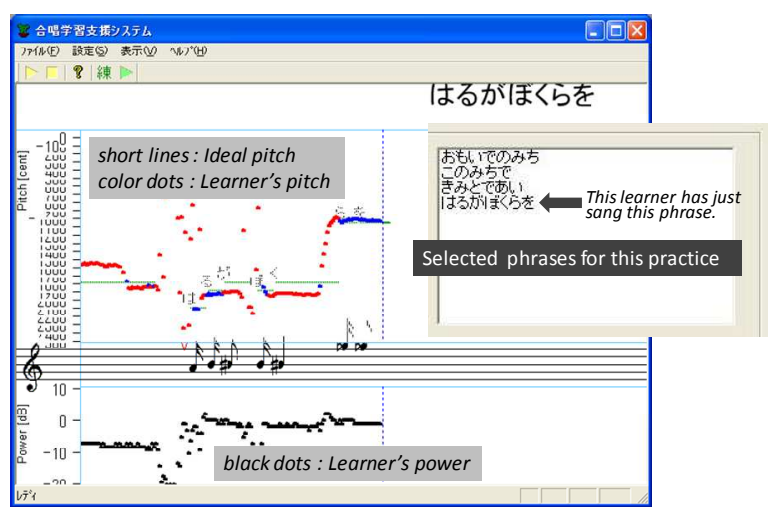

Figure 10. An example screen image of the system interface for phrase practice.

\section{System Evaluation}

\subsection{Whole Singing Support}

In this section, we examine the relationship between the real-time/post instruction from our system and the improvement in the singing voice. Not only the song which is taught by the master (YORU NO UTA, explained in 3.2), but also a song not taught (Song name: TEGAMI, Music and Lyrics: Anjera Aki, Arrangement: Ohta Sakurako) is used for this experiment. By using a song not taught, the versatility of this system is also examined.

The users of the learning system are all novices, who had never been taught by the master. The subjects are divided into 3 groups. Each group is given different methods of use.

Group A: use real-time instruction and post instruction.
Group B: use real-time instruction.

Group C: do not use any instruction function of our system.

Each member sings two songs using these two auditory feedbacks (FB in short).

FB1: the synthesized voice in the singer's part.

FB2: the synthesized voices in all parts.

FB1 simulates the singing by one part, and FB2 simulates the singing by all parts. Under these conditions, each subject sings each song 10

times. In addition, for Groups A and B, a questionnaire about the instructions from our system was presented after singing each time.

Table1 shows the number of subjects who are able to improve their singing. (a) is the song taught, and (b) is the song not taught by the master. For both songs, more than half of the subjects could improve their singing in terms of pitch, but improvement for Group $\mathrm{C}$ could not be confirmed. In terms of power, the effectiveness of the system is less certain. There was no difference between the three groups. For the song not taught, Groups A and B show considerable evidence of improvement for both FB1 and FB2. From the comparison with Group $\mathrm{C}$ and other groups, the accuracy of pitch in the singing voice is clearly improved by using our instruction. Moreover, when the users practice the song not taught with our system, the effectiveness for the improvement in pitch accuracy is confirmed for both feedback conditions.

Table 1. Comparison of the number of the subjects for whom the evaluation result of the song improved

(a) song taught

(b) song not taught

\begin{tabular}{|c|c|c|c|c|c|c|c|c|c|}
\hline \multirow{3}{*}{ Group } & \multicolumn{2}{|c|}{ Pitch } & \multicolumn{2}{|c|}{ Power } & & \multicolumn{2}{|c|}{ Pitch } & \multicolumn{2}{|c|}{ Power } \\
\hline & \multicolumn{2}{|c|}{ FB } & \multicolumn{2}{|c|}{ FB } & \multirow{2}{*}{-Group } & \multicolumn{2}{|c|}{ FB } & \multicolumn{2}{|c|}{ FB } \\
\hline & 1 & 2 & 1 & 2 & & 1 & 2 & 1 & 2 \\
\hline A & 4 & 2 & 1 & 1 & A & 2 & 3 & 1 & 0 \\
\hline B & 2 & 3 & 1 & 0 & B & 2 & 2 & 2 & 0 \\
\hline $\mathrm{C}$ & 1 & 1 & 2 & 0 & $\mathrm{C}$ & 0 & 0 & 0 & 0 \\
\hline
\end{tabular}

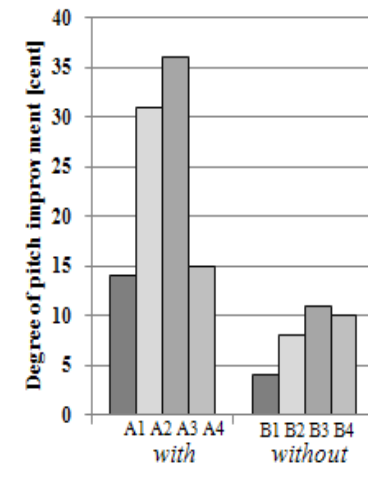

(a) Improvement in Pitch

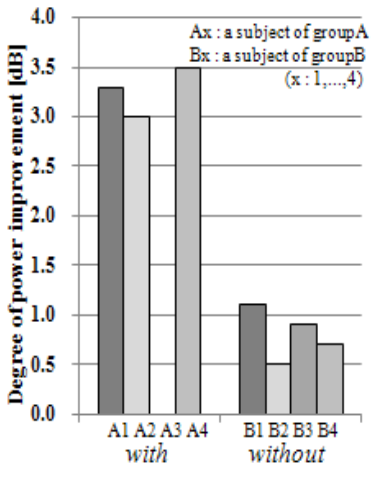

(b) Improvement in Power 
Figure 11. Degree of improvement in pitch (on the left) and power (on the right) with/without instruction words.

By comparing with Groups A and B, especially focusing on the instruction words in advice given by the system, we consider the advantages of the post instruction function. Fig.11 shows the degree of improvement in pitch (a) and power (b) with and without instruction words, when the subjects sing the song taught with FB2. In this Figure, "with" means Group A (A1 shows one subject in Group A), and "without" means Group B. Group A shows a distinct improvement over Group B. By the Student t-test, the significant differences of the improvements of both groups are 3\% with FB1 and 1\% with FB2. For the song not taught, the significant differences are $1 \%$ with FB1 and FB2. These improvements were confirmed by the occurrence of the overshoot phenomenon in voice power.

\subsection{Phrase Singing Support}

We examine the verification of the pitch improvement using this function. In this experiment, we used a new chorus song for practice. This song is different from the songs used in the experiments described in 3.2 and 5.2. Two subjects (Subject-A, Subject-B) participated in this experiment. Subject-A used our new system which has a phrase practice function. Subject-B used our old system which does not have a phrase practice function. Subjects were followed for four days of practice. We instructed them to limit one practice to a maximum of 30 minutes. Each subject practiced 12 times in this experiment. We tried to analyze the continuous change of the following two items.

(1) The number of off-pitch notes which have more than plus or minus 25 [cents] difference from the ideal pitch.

(2) The average offset of the pitch in a singing phrase.

Fig.12 shows the results of (1), and Fig.13 shows the results of (2). The horizontal axis in both graphs is the practice time. Both subjects improved their pitch offset as a result of the experiment. We want to express the degree of improvement as the gradient of a linear approximation for all data for each subject. For the first item (shown in Fig.12), the value of Subject-A is 2.62 and Subject-B is 1.12. For the second item (shown in Fig.13), Subject-A is 0.71 and Subject-B is 0.72 . Especially for the first item, "the number of off-pitch notes which have more than plus or minus 25 [cents] difference from the ideal", Subject-A shows a more remarkable improvement in singing pitch. Thus, we can show the effectiveness for phrase practice by using this phrase practice support function.

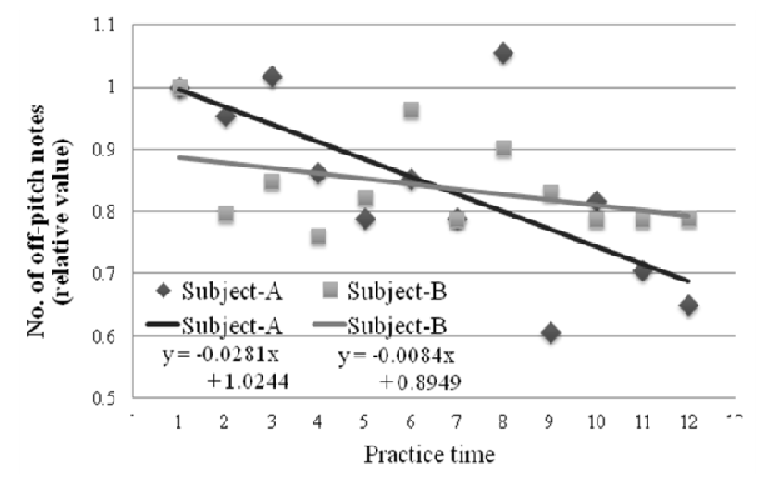

Figure 12. The number of off-pitch notes.

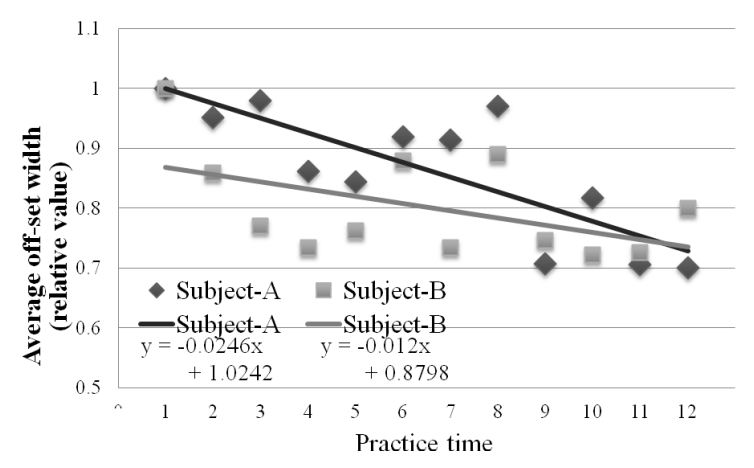

Figure 13. The average offset width.

\section{Conclusion}

The purpose of this study is to explore a choral learning support environment which supports individual choir practice. The proposed system is able to simulate the other members of a real chorus group by using synthesized voices. This system has real-time and post instruction functions based on the three types of expertise which were extracted from a chorus leader. By using these functions, we can provide an effective, efficient and adaptive chorus learning environment.

To extract the leader's knowledge about rhythm and phonetic value is our next step. We also want to gather more instruction words and analyze the relationship between these words and the acoustical features of voices.

\section{Acknowledgements}

This work was supported by JSPS KAKENHI Grant Number 24650551.

\section{References}

[1] K. Furukawa, "Skill Science", Journal of Japanese Society Artificial Intelligence, vol.19, no.3, pp.355-364, 2004.

[2] European Commission, Joint Research Centre, Institute for Prospective Technological Studies, The Information Society Unit : http://is.jrc.ec.europa.eu/pages/EAP/eLearning.html

[3] The Learning and Skills Group : 
http://learningandskillsgroup.ning.com/

[4] T.Nagai,M.Kayama and K.Itoh : "A Basic Study on a Drawing-Learning Support System in the Networked Environment", Human-Computer Interaction. Novel Interaction Methods and Techniques (Lecture Notes in Computer Science Volume 5611),pp.860-868, 2009.

[5] S.Moria,Y.Ohtanib and K.Imanakaa : "Reaction times and anticipatory skills of karate athletes", Human Movement Science, vol.21, issue 2, pp.213-230 2002.

[6] M.Suwa : "Metacognitive Verbalization as a Tool for Acquiring Embodied Expertise", Journal of Japanese Society for Artificial Intelligence, vol.20, no.5, pp.525-532, 2005.

[7] T. Muratani, and S.Sekiya, "Chorus Handbook", Tokyo:Nishida Syoten, 1980.

[8] H. Takeuchi, "Practical Situations and Management methods of Chorus Traning", Tokyo: Ongaku no tomo sha Corp., 1992.

[9] BERA Music Education Review Group, Mapping Music Education in the UK, http://www.bera.ac.uk/files/reviews/musicreview.pdf, 2001. unpublished.

[10] D. M. Howard, and G. F. Welch, Microcomputer-based singing ability assessment and development, Applied Acoustics, vol.27, pp.89-102, 1989.

[11] D Hoppe, M. Sadakata and P. Desain, Development of real-time visual feedback assistance in singing training : a review, Journal of Computer Assisted Learning, vol.22, pp.308-316, 2006.

[12] T. Nakano, M. Goto, and Y. Hiraga, MiruSinger: A singing skill visualization interface using real-time feedback and music CD recordings as referential data, Proc. of the 9th IEEE Int. Symp. on Multimedia Workshops, pp.75-76, 2007.

[13] KAWAI Musical Instruments Mfg. Co. Ltd., "Primavista2", htp://www.kawai.co.jp/cmusic/products/pv/index.htm, 2007, unpublished.

[14] M.Kayama, K. Itoh, K.Asanuma, M. Hashimoto and M. Otani, "A Chorus Learning Support System Based on the Tutoring Knowledge of the Chorus Leader", Proc. the 10th IEEE International Conference on Advanced Learning Technology(ICALT), pp.11-15 2010.

[15] M.Kayama, K. Itoh, K. Asanuma, M. Hashimoto and M. Otani, "Instructional Expertise for Phrase Singing and its Application for a Chorus Learning Support System", Proc. of the 11th IEEE International Conference on Advanced Learning Technology(ICALT), pp.323-325, 2011.

[16] W. Hoppers, "Non-formal education and basic education reform : a conceptual review", NESCO Int., Inst., for Educational Planning, http://unesdoc.unesco.org/images/0014/001444/144423e.pdf, 2006, unpublished.

[17] Cypton Future Media, Inc., "VOCALOID2", http://www.crypton. co.jp/mp/pages/prod/vocaloid/cv01.jsp, 2007, unpublished. 